\title{
Efficacy and Safety of Guardcel Nasal Packing After Endoscopic Sinus Surgery: A Prospective, Single- Blind, Randomized Controlled Study
}

\author{
Byungjin Kang ${ }^{1} \cdot$ Jeong-Rok Kim¹ $\cdot$ Jae-Min Shin ${ }^{1} \cdot$ II-Ho Park ${ }^{1} \cdot$ Heung-Man Lee Ka,3 $^{1,2}$ \\ ${ }^{1}$ Department of Otorhinolaryngology-Head and Neck Surgery and ${ }^{2}$ Division of Brain Korea 21 Program for Biomedical Science, Korea University \\ College of Medicine, Seoul; ${ }^{3}$ In vitro Diagnostic Medical Devices Support Center, Korea University Guro Hospital, Seoul, Korea
}

Objectives. Nasal packing after endoscopic sinus surgery is frequently used to control postoperative bleeding, enhance the wound healing process, and prevent lateralization of the middle turbinate, which causes insufficient ventilation. Many biodegradable materials have been developed to reduce pain and mucosal damage during packing removal. The purpose of this study was to compare the efficacy of Guardcel (Genewel Co.) middle meatal packing with a traditional nonabsorbable middle meatal packing, Merocel (Medtronic Xomed), on wound healing and patient satisfaction.

Methods. In this prospective, single-blind, randomized controlled study, we enrolled 32 consecutive patients (64 nostrils) undergoing bilateral endoscopic sinus surgery at Korea University Guro Hospital from February 2015 to August 2015. Guardcel and Merocel were inserted postoperatively into a randomly assigned side. Objective findings about bleeding, hemostasis, adhesion, and infection were evaluated with nasal endoscopy. Patients' symptoms including pain and nasal obstruction were evaluated with a visual analog scale. Each evaluation was done at 2-3 days, 1 week, 2 weeks, and 4 weeks after surgery.

Results. At 2-3 days after endoscopic sinus surgery, the Guardcel side had a significantly less hemostasis time than the Merocel side $(P=0.001)$. During this period, the pain during packing removal was significantly lower on the Guardcel-inserted side than the Merocel-inserted side $(P=0.002)$. At two weeks after surgery, the adhesion score on the Guardcel side was significantly lower than that of the Merocel side $(P=0.011)$. Other parameters during the study follow-up periods were not statistically significant. There were no severe adverse reactions.

Conclusion. Guardcel, a newly developed packing material, appeared to shorten the hemostasis time and reduce pain sensation at 2-3 days after surgery; it also prevented adhesion formation 2 weeks after surgery when compared with the control. Guardcel can be an effective and safe candidate to replace conventional packing materials after endoscopic sinus surgery.

Keywords. Nasal Packing; Absorbable Packing; Hemostasis; Adhesion; Endoscopic Sinus Surgery

\section{INTRODUCTION}

- Received July 22, 2016

Revised October 12, 2016

Accepted October 12, 2016

- Corresponding author: Heung-Man Lee

Department of Otorhinolaryngology-Head and Neck Surgery, Korea

University Guro Hospital, Korea University College of Medicine,

148 Gurodong-ro, Guro-gu, Seoul 08308, Korea

Tel: +82-2-2626-3185, Fax: +82-2-868-0475

E-mail: lhman@korea.ac.kr
Chronic rhinosinusitis is the most common inflammatory diseases in rhinology. In the USA, up to $16 \%$ of the adult population suffer from this condition [1]. The prevalence of chronic rhinosinusitis in Korea was $6.95 \%$ in a previous study [2]. Chronic rhinosinusitis results in various symptoms including nasal obstruction, purulent rhinorrhea, facial pain, headache, chronic cough, and hyposmia. These symptoms have a negative effect on patient quality of life.

Copyright (C) 2017 by Korean Society of Otorhinolaryngology-Head and Neck Surgery.

This is an open-access article distributed under the terms of the Creative Commons Attribution Non-Commercial License (http://creativecommons.org/licenses/by-nc/4.0)

which permits unrestricted non-commercial use, distribution, and reproduction in any medium, provided the original work is properly cited. 
Endoscopic sinus surgery (ESS) is generally accepted as the gold standard surgical treatment of chronic rhinosinusitis [3]. Surgery aims to restore drainage and ventilation via the ostium of each sinus and to remove pathologic mucosa with minimal damage to normal mucosa. Appropriate postoperative care is mandatory to improve surgical outcomes and reduce patient discomfort.

After ESS, nasal packings are frequently used despite debate regarding their necessity. The purpose of nasal packing is to control postoperative bleeding, enhance the wound healing process, and prevent adhesion and lateralization of the middle turbinate, which can cause insufficient ventilation [4]. Conventional packing materials such as Merocel (polyvinyl acetate sponge, Medtronic Xomed, Jacksonville, FL, USA) are widely used and have several advantages including cost, sufficient support ability, and ease of manipulation. However, they can cause patient discomfort, such as nasal obstruction and pain, and cause mucosal damage and bleeding when removed [5].

Many biodegradable or absorbable materials have been developed to remedy these shortcomings of conventional packing materials. These products vary in composition, mechanism of action, method of delivery and clearance, and cost. Guardcel (Genewel Co., Seongnam, Korea) is a newly developed biodegradable packing material, composed of a mixture of collagen, hyaluronic acid, and carboxymethylcellulose (Fig. 1). Hyaluronic acid helps keep the surgical site moist, reduces adhesions, and
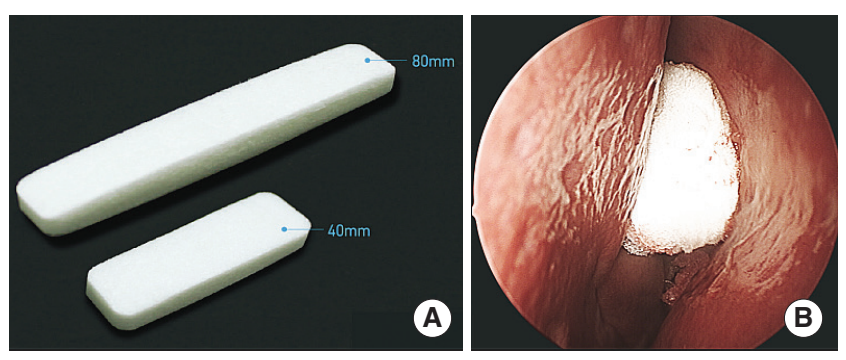

Fig. 1. (A) Gross shape of Guardcel (Genewel Co.) and (B) endoscopic findings in the left middle meatus after Guardcel packing at the conclusion of surgery.

\section{H I}

- The efficacy of Guardcel middle meatal packing was compared to that of Merocel.

- The pain during packing removal was lower on the Guardcel side than the Merocel side.

- At the 2nd week visit, the Guardcel side had less adhesion than the Merocel side.

- There were no significant infections or severe adverse reaction in both groups.

- Guardcel is an effective and safe packing material after endoscopic sinus surgery. decreases healing time [6]. Carboxymethylcellulose is a polysaccharide foam that actively promotes platelet aggregation [5]. It is not absorbable, but it can be flushed out of the nose by saline irrigation, eliminating the need for extraction. Therefore, we anticipate that this new packing material may have advantages due to the use of these ingredients.

The purpose of this study was to compare the efficacy of Guardcel middle meatal packing and a traditional nonabsorbable middle meatal packing, Merocel, on wound healing and patient satisfaction.

\section{MATERIALS AND METHODS}

\section{Subjects}

We enrolled 32 consecutive patients (64 nostrils) undergoing ESS at Korea University Guro Hospital, Seoul, Korea in a prospective, single-blind, randomized controlled study between February 2015 and August 2015. Inclusion criteria were age between 19 and 70, bilateral chronic rhinosinusitis requiring surgery, and a difference of 2 or less in the Lund-MacKay computed tomography (CT) scan score between the sides. Exclusion criteria were unilateral disease, massive sinonasal polyposis, history of previous sinus surgery, and other underlying diseases that may affect outcomes. Ethics approval was obtained from the Korea University Medical Center Institutional Review Board (IRB no. MD14035) and informed consent was obtained from all patients before enrollment.

\section{Study design}

As the difference of Guardcel (Genewel Co.) and Merocel (Medtronic Xomed) appearance is evident, we proceeded the study as a single-blinded model. At the time of the enrollment, patients were randomized according to computer-generated random numbers to determine which side was to receive Guardcel packing intraoperatively. The other side was packed with Merocel packing within the middle meatus. Each packing materials were alike in size. All patients underwent ESS bilaterally under general anesthesia, and all surgeries were performed by a single surgeon (HML) using similar methods. After surgery, each packing was inserted into the appropriate side. The patients were blinded to which side had the Guardcel packing and which side had the control packing. We evaluated an efficacy of the Guardcel by means of degrees of bleeding, hemostasis, adhesion, infection, and symptoms about pain and nasal obstruction.

Depending on the protocol of Korea University Guro Hospital, patients underwent removal of packing at 2 or 3 days after surgery. At that time, Merocel was removed entirely and degraded packing of Guardcel was sucked out partially. Intranasal crusts in both side were removed carefully. Then, patients were asked to give a score from 0 (no symptoms) to 10 (maximal symptoms) using the visual analog scale (VAS), about the degrees of pain before removal of packing, pain during removal of 
packing, and nasal obstruction on each side. During removal, bleeding and time to hemostasis were scored from 0 to 3 by a nonblinded surgeon (HML). The grade of adhesion and infection were retrospectively evaluated with nasal endoscopic photographs by two independent rhinologists (JMS and IHP). Table 1 shows the criteria for grading score of bleeding, hemostasis, adhesion, and infection. Patients were scheduled to revisit the clinic at 1, 2, and 4 weeks postoperatively (Fig. 2). During these visits, the same objective scoring criteria were evaluated, and patients were scored for nasal obstruction and asked about possible adverse reactions. The protocol of the trial is summarized in the Consolidated Standards of Reporting Trials (CONSORT) flow diagram (Fig. 3).

The primary outcome of this study was defined as a difference between the efficacy on hemostasis of Guardcel and that of Merocel at 2-3 days after surgery. Secondary outcomes included other measured variables during the investigation period. To evaluate the safety of each agent, all abnormal symptoms or signs and abnormal laboratory or imaging results collected during the investigation period were analyzed. We expected causal adverse reactions including pain, infection and allergic reactions. The vital signs, complete blood count, routine blood chemistry, urine analysis, an electrocardiogram, and a chest X-ray were obtained for each subject prior to the operation as well as at 4 weeks after surgery.

Table 1. Scales used to evaluate objective findings about bleeding, hemostasis, adhesion, and infection

\begin{tabular}{|c|c|}
\hline Criteria & Score \\
\hline \multicolumn{2}{|l|}{ Bleeding } \\
\hline No bleeding & 0 \\
\hline Minimal (confined to nasal cavity) & 1 \\
\hline Moderate (bleeding out of nasal cavity) & 2 \\
\hline Needed packing again & 3 \\
\hline \multicolumn{2}{|l|}{ Hemostasis } \\
\hline Required time $<5$ min & 0 \\
\hline $5 \leq$ Required time $<10 \mathrm{~min}$ & 1 \\
\hline $10 \leq$ Required time $<20 \mathrm{~min}$ & 2 \\
\hline Required time $\geq 20 \mathrm{~min}$ & 3 \\
\hline \multicolumn{2}{|l|}{ Adhesion } \\
\hline No adhesion & 0 \\
\hline $\begin{array}{l}\text { Visible adhesion, no interference with sinus ventilation } \\
\text { and drainage }\end{array}$ & 1 \\
\hline $\begin{array}{l}\text { Visible adhesion, mild interference with sinus ventilation } \\
\text { and drainage }\end{array}$ & 2 \\
\hline $\begin{array}{l}\text { Total adhesion between middle turbinate and lateral } \\
\text { nasal wall (needed adhesiolysis) }\end{array}$ & 3 \\
\hline \multicolumn{2}{|l|}{ Infection } \\
\hline No evidence of infection & 0 \\
\hline Scanty mucopurulent discharge and mild edema & 1 \\
\hline $\begin{array}{l}\text { Gross mucopurulent discharge and edema with } \\
\text { extinction of ethmoid cavity }\end{array}$ & 2 \\
\hline $\begin{array}{l}\text { Profuse mucopurulent discharge and polyposis } \\
\text { (needed treatment) }\end{array}$ & 3 \\
\hline
\end{tabular}

\section{Statistical analysis}

Sample size was calculated based on previously published literature that compare the efficacy of Nasopore (Biodegradable syn-

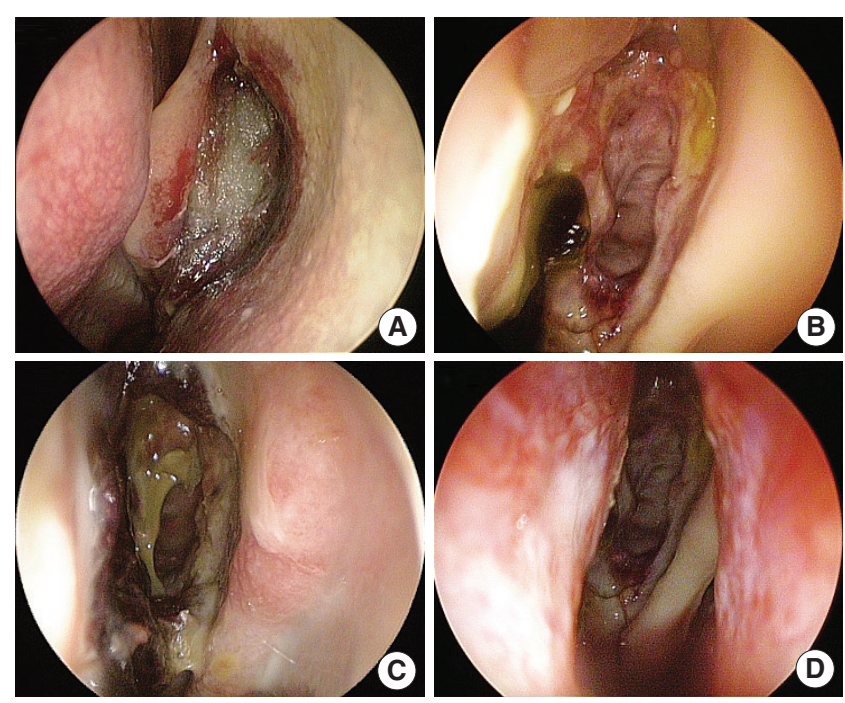

Fig. 2. Follow-up endoscopic findings. Images of left middle meatus which side Guardcel (Genewel Co.) was inserted to $(A)$ one week after surgery. Degraded packing material was remained. (B) Four weeks after surgery. And images of right middle meatus which side Merocel (Medtronic Xomed) was inserted to, (C) one week after surgery. Lots of crusts were noted. Packing material had been removed at 2-3 day after surgery. (D) Four-weeks after surgery.

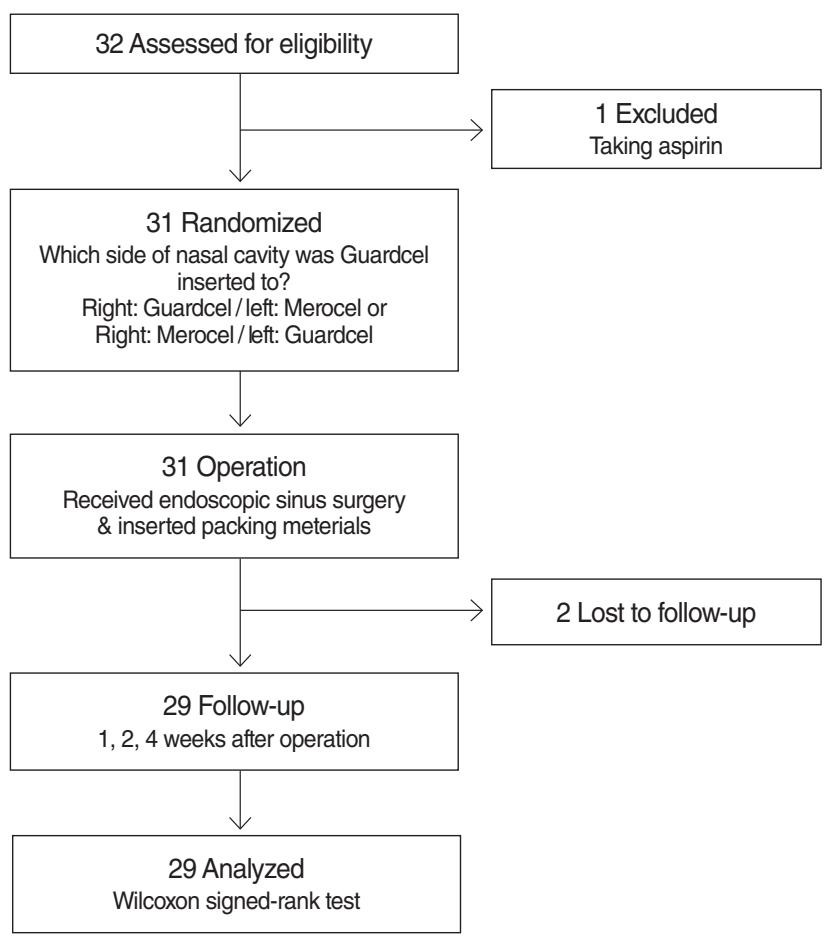

Fig. 3. Consolidated Standards of Reporting Trials (CONSORT) diagram of the randomized controlled trial. 
thetic polyurethane foam, Polyganics BV, Groningen, Netherlands) to that of Merocel, with $15 \%$ dropout rate [7]. Statistical analysis was performed using IBM SPSS ver. 23.0 (IBM Co., Armonk, NY, USA). The parameters were compared using the Wilcoxon signed-rank test. A $P$-value $\leq 0.05$ was considered statistically significant.

\section{RESULTS}

\section{Patient demographics and Lund-Mackay CT scores}

Thirty-two patients were included in this study. The mean age was 45 years (range, 22 to 66 years). Twenty-four patients were males $(75 \%)$. The Guardcel packing material was randomly assigned to the left nasal cavity in 21 patients $(66 \%)$. The mean preoperative Lund-MacKay CT score on the side which received Guardcel packing was 6.9 (range, 3 to 11), while the mean score for the Merocel packing was 6.8 (range, 3 to 11). No patient had a score difference exceeding \pm 2 between each side. During the study, two patients dropped out because they wanted to discontinue the study. One patient was excluded due to taking aspirin. Therefore, the data from 29 patients were analyzed (Table 2).

\section{Objective parameters}

At 2 or 3 days after surgery, the Guardcel side had a significantly shorter hemostasis time (mean score, 0.76 vs. $1.41 ; P=0.001$ ) (Fig. 4). There was no difference in bleeding, adhesion, or infec-

Table 2. Subject demographics $(n=32)$

\begin{tabular}{lc}
\hline Demographic & Value \\
\hline Sex & \\
Male & 24 \\
Female & 8 \\
Age (yr) & $45.2 \pm 12.8$ \\
Lund-MacKay computed tomography score & \\
$\quad$ Right & $6.9 \pm 2.1$ \\
Left & $6.8 \pm 2.1$ \\
\hline
\end{tabular}

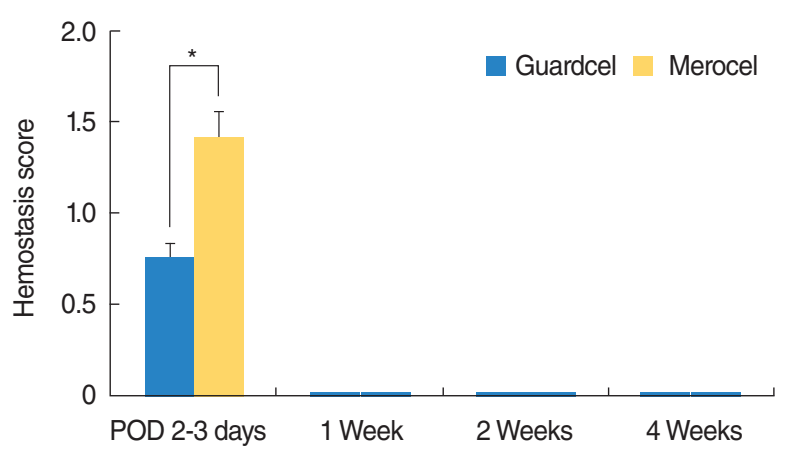

Fig. 4. Comparison of the hemostasis time between Guardcel (Genewel Co.) and Merocel (Medtronic Xomed) groups on each postoperative day (POD). At 2 or 3 days after surgery, the Guardcel side had significantly shorter hemostasis times $\left({ }^{\star} P<0.05\right)$. tion at that time. At the 1st week visit after surgery, there was no significant difference in any parameters. At the 2 nd week visit, the Guardcel-inserted side had significantly less adhesion than the Merocel-inserted side (mean score, 0.24 vs. $0.72 ; P=0.011$ ) (Fig. 5). However, this difference disappeared at the 4th week. The other examined parameters had no statistically significant differences during the postoperative follow-up periods.

\section{Subjective parameters}

All 29 patients completely answered the VAS about pain before packing removal, pain during packing removal, nasal congestion, and nasal obstruction during the follow-up period. At 2 or 3 days after surgery, the side with Guardcel packing had significantly lower pain scores during packing removal than the side with Merocel packing (mean, 4.41 vs. $6 ; P=0.002$ ). However, there was no significant difference in pain before packing removal (mean score, 2.90 vs. 3.17; $P=0.230$ ) and nasal obstruction (mean score, 4.34 vs. $4.66 ; P=0.084$ ) between the Guardcel packing side and the Merocel packing side (Fig. 6). During fol-

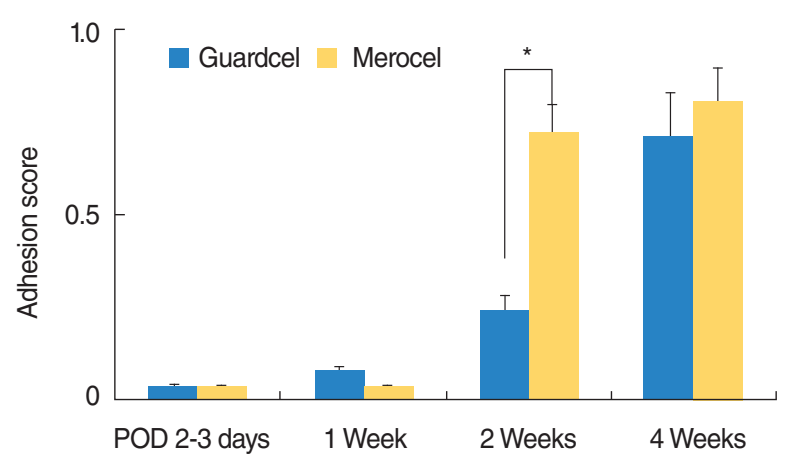

Fig. 5. Comparison of the grade of adhesion between Guardcel (Genewel Co.) and Merocel (Medtronic Xomed) groups on each postoperative day (POD). At the 2nd week visit, the Guardcel side had significantly less adhesion than the Merocel side $\left({ }^{*} P<0.05\right)$. There was no statistically significant difference in adhesion at other times between both groups.

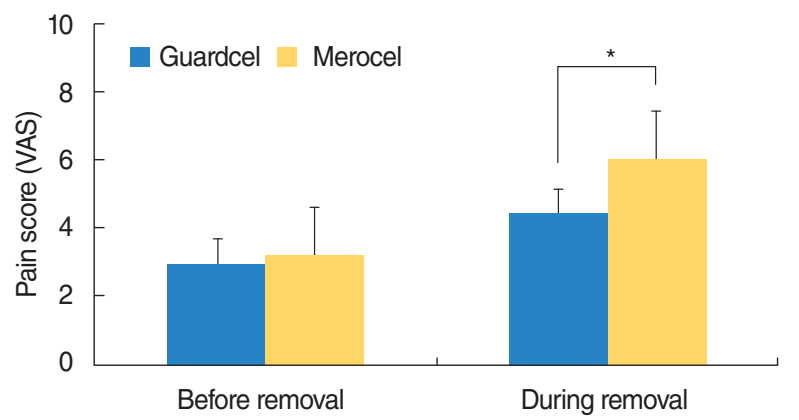

Fig. 6. Subjective pain scores before and during removal of packing 2-3 days after surgery. The side with Guardcel (Genewel Co.) packing had significantly lower pain scores during packing removal than the side with Merocel (Medtronic Xomed) packing $\left({ }^{\star} P<0.05\right)$. There was no statistically significant difference in the pain scores before packing removal between both groups. VAS, visual analog scale. 


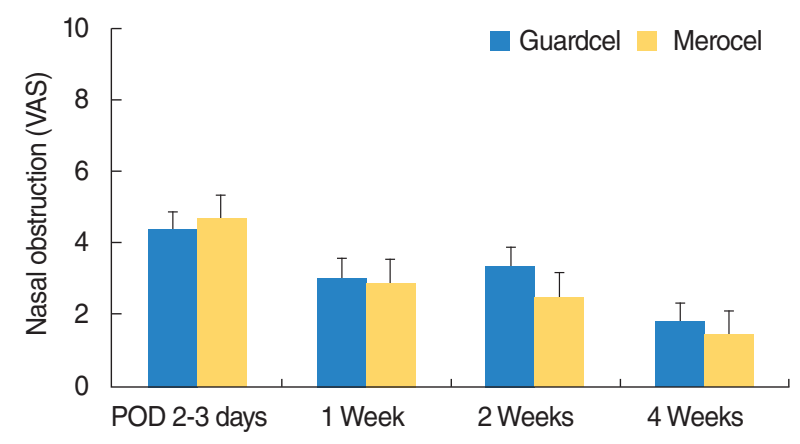

Fig. 7. Changes in nasal obstruction symptoms after endoscopic sinus surgery. There was no statistically significant difference in nasal obstruction symptoms between both groups. VAS, visual analog scale; POD, postoperative days.

low-up visits at the 1st, 2nd, and 4th weeks after surgery, there were no statistically significant differences in nasal obstruction between both sides (Fig. 7).

\section{Adverse reactions}

Three patients had a fever and a patient each had skin rash and abdominal pain postoperatively. However, there were no possible adverse reactions associated with packing materials in either group. Laboratory findings and imaging results, between before the surgery and postoperative 4th week, did not show the clinically significant change in all patients.

\section{DISCUSSION}

The use of middle meatal packing after ESS is common among surgeons, despite the debate regarding the necessity of packing. However, removal of nasal packing after ESS may be the most unpleasant experience for patients during the treatment term, including surgery. In addition, inappropriate use of nasal packing and possible trauma during packing removal are known to impede the mucosal healing processes [8]. Therefore, many biodegradable materials have been developed and are widely used after ESS.

Guardcel is composed of a mixture of collagen, hyaluronic acid, and carboxymethylcellulose. In a previous study, Berlucchi et al. [6] reported that MeroGel (Hyaluronic acid) is safe and well-tolerated and seems to decrease synechia formation when compared to polyvinyl acetate packing. In addition, Leunig et al. [9] and Kastl et al. [10] reported that Rapid Rhino Sinus dressing (carboxymethylcellulose packing) did not increase subjective pain of patients, compared to no nasal packing. However, they failed to find other advantages when using carboxymethylcellulose packing. Guardcel has characteristics of both hyaluronic acid and carboxymethylcellulose. In our study, patients had less pain and less bleeding at 2-3 days postoperatively, and there were fewer adhesions at 2 weeks after surgery on the
Guardcel side. There were no significant infections or reports about severe adverse reactions. In addition, Shoman et al. [7] reported that Nasopore (synthetic polyurethane foam) does not significantly reduce patient discomfort compared to Merocel. Their results suggested significantly slower mucosal healing in the Nasopore group than in the Merocel group, but this effect disappeared after 3 months postoperatively [7]. Similarly, Guardcel did not show a long-term difference compared to Merocel in our study.

In this prospective, single-blinded, randomized controlled study, we compared the efficacy of wound healing and patient satisfaction when using a newly developed biodegradable packing, Guardcel, to that when using conventional nonabsorbable packing material (Merocel). As the primary endpoint, the time to hemostasis after packing removal was significantly lower on the Guardcel side. However, hemostasis score of both groups were favorable after 1 week postoperatively. Subjective pain during packing removal was also significantly lower on the Guardcel side. When patients visited the clinic for a second follow-up, the objective adhesion score was better on the Guardcel side. There was no patient requiring an adhesiolysis in both groups. All other parameters investigated during the studied period had no statistically significant differences on both sides. These findings suggest that Guardcel may reduce time to hemostasis and pain during packing removal and prevent short-term postoperative adhesion.

However, these findings did not persist until the 4th followup. Bleeding and time of hemostasis make problems mainly during immediate postoperative period, and the scores of hemostasis in this study were all reported as zero in both groups after 1 week postoperatively. We failed to proof the difference of the scores of adhesion and nasal obstruction at 4th week between both group, and the results left much to be desired. Hyaluronic acids have a relatively short half-life less than 3 days in vitro. We thought that a mixed composition with carboxymethylcellulose may help this shortcoming, because of its nonabsorbable nature. However, they might not have a long-term effect. The previous studies were also showed variable results. Wormald et al. [11] reported that MeroGel have no significant benefit in terms of synechia, edema and infection. Berlucchi et al. [6] reported that MeroGel have an effect to reduce the synechia compared to control, at 4th and 12th weeks follow-up. These differences including our results are difficult to explain, however, review of previous literature suggests that postoperative care including saline irrigation and endoscopic debridement may contribute their results [7]. We performed an endoscopic debridement of crusts during follow-up period, and remaining degraded materials of Guardcel were routinely removed at 1st week, because of concerns about infections. Longer follow-up period might be needed to prove any difference of long-term outcomes between both groups. Nevertheless, it is important that there was no significant harmful effect on healing in Guardcel side compared to 
control.

This pilot study has several limitations. A small population was included the study, and longer follow-up periods are required to investigate the any significant longer-term effect of these materials. And this study was conducted with a singleblind setting. Because Guardcel and Merocel have different shape and form, it was not possible to blind the surgeon about the packing. However, the allocation of Guardcel and control was informed to surgeon just before insertion using a sealed randomization envelope, in order to prevent biases, like the Barnum effect. Also, recall bias could have affected the results when patients answered the questionnaires after the first followup. Subsequent studies should be conducted with a large population and double-blind settings comparing to other absorbable packing materials.

In conclusion, a newly developed packing material, Guardcel, appears to reduce pain and shorten the hemostasis time 2-3 days after ESS. We also observed less adhesion formation 2 weeks after surgery when Guardcel was used. However, there were no significant differences at 4 weeks after surgery in any parameters. Guardcel can be a safe candidate for replacing conventional packing materials after ESS.

\section{CONFLICT OF INTEREST}

No potential conflict of interest relevant to this article was reported.

\section{ACKNOWLEDGMENTS}

This study was supported by a grant from the Korean Health Technology R\&D Project, Ministry of Health \& Welfare (No. HI13C1501), Sejong, Korea (by Heung-Man Lee).

\section{REFERENCES}

1. Benninger MS, Ferguson BJ, Hadley JA, Hamilos DL, Jacobs M, Kennedy DW, et al. Adult chronic rhinosinusitis: definitions, diagnosis, epidemiology, and pathophysiology. Otolaryngol Head Neck Surg. 2003 Sep;129(3 Suppl):S1-32.

2. Kim YS, Kim NH, Seong SY, Kim KR, Lee GB, Kim KS. Prevalence and risk factors of chronic rhinosinusitis in Korea. Am J Rhinol Allergy. 2011 May-Jun;25(3):117-21.

3. Govindaraj S, Adappa ND, Kennedy DW. Endoscopic sinus surgery: evolution and technical innovations. J Laryngol Otol. 2010 Mar; 124(3):242-50.

4. Valentine R, Wormald PJ, Sindwani R. Advances in absorbable biomaterials and nasal packing. Otolaryngol Clin North Am. 2009 Oct; 42(5):813-28.

5. Yan M, Zheng D, LiY, Zheng Q, Chen J, Yang B. Biodegradable nasal packings for endoscopic sinonasal surgery: a systematic review and meta-analysis. PLoS One. 2014;9(12):e115458.

6. Berlucchi M, Castelnuovo P,Vincenzi A, Morra B, Pasquini E. Endoscopic outcomes of resorbable nasal packing after functional endoscopic sinus surgery: a multicenter prospective randomized controlled study. Eur Arch Otorhinolaryngol. 2009 Jun;266(6):839-45.

7. Shoman N, Gheriani H, Flamer D, Javer A. Prospective, doubleblind, randomized trial evaluating patient satisfaction, bleeding, and wound healing using biodegradable synthetic polyurethane foam (NasoPore) as a middle meatal spacer in functional endoscopic sinus surgery. J Otolaryngol Head Neck Surg. 2009 Feb;38(1):112-8.

8. Mo JH, Han DH, Shin HW, Cha W, Chang MY, Jin HR. No packing versus packing after endoscopic sinus surgery: pursuit of patients' comfort after surgery. Am J Rhinol. 2008 Sep-Oct;22(5):525-8.

9. Leunig A, Betz CS, Siedek V, Kastl KG. CMC packing in functional endoscopic sinus surgery: does it affect patient comfort? Rhinology. 2009 Mar;47(1):36-40.

10. Kastl KG, Betz CS, Siedek V, Leunig A. Effect of carboxymethylcellulose nasal packing on wound healing after functional endoscopic sinus surgery. Am J Rhinol Allergy. 2009 Jan-Feb;23(1):80-4.

11. Wormald PJ, Boustred RN, Le T, Hawke L, Sacks R. A prospective single-blind randomized controlled study of use of hyaluronic acid nasal packs in patients after endoscopic sinus surgery. Am J Rhinol. 2006 Jan-Feb;20(1):7-10. 